\title{
Research on Prime Circuit With High Sensitivity for Photoelectric Detection Based on Pgtia
}

\author{
Qi-Chao SONG ${ }^{a^{*}}$, Hai-Cheng LIU, Hui GAO, Yan-Ping LI, Peng ZHANG, \\ Guo-Ping SHAO, Hong-Xia CHU
}

College of electrical and information engineering, Heilongjiang institute of technology, Harbin, China

asqch@163.com

${ }^{*}$ Corresponding author

Keywords: Transimpedance Amplifier, Photodiode, Sensitivity, Analog Switches.

\begin{abstract}
Photoelectric sensor is widely used in the fields of the measurements of fiber sensor and the chemical parameters. Photoelectric detection circuit based on the photodiode is constructed with transimpedance amplifier. The precision and sensitivity of circuit are adjusted by programmable gain. The index requirements of the amplifier are clear, and the feasible circuit is given at last.
\end{abstract}

\section{Introduction}

Basis for the requirement of precision and weak and the actual situation, precise signal detection system based on amperometric sensoror photodiode always need high sensitivity transimpedance amplifier (TIA) and programmable gain to furthestimprove the detection dynamic range.

The essence of TIA is the current/voltage converter (I/V) which converts current signal to voltage signal. Resistance is the simplest I/V circuit. On the basis of Ohm's law, the current flowing on the resistance will yield pressure drop. When the weak sensitivity is measured or bigger sensitivity is need, the value of resistance must be bigger to get bigger voltage output. Generally, the resistance with big resistance value has low pricision and high noise, and the resistance with high load combines with the input capacitance of rear stage circuit can product a bigger time constant RC, so the high frequency response of the system will be affected and the dynamic range will be decreased.In addition, I/V circuit which is the prime circuit of the detection system is crucial to realize high sensitivity and high resolution of the whole detection system. In the research of weak signal, rear stage need amplification and filter, and the amplification factor are hundreds to thousands times, if the precise design is not good, the signal and the noise are amplified. So the amplifier must be used, and the characteristic of virtual open is used to construct the TIA to realize the I/V convert of current signal with weak or high sensitivity. Programmable gain transimpedance amplifier (PGTIA) with wide bandwidth and high sensitivity is designed based on photoelectric detection by using photodiode.

\section{Photoelectric Detection Circuit Based on the Photodiode}

Photodiode is a device which converts optical signal to weak current signal, and it is widely used in the fields of fiber communication and sensor. When photodiode works under the reverse bias (or zero bias), current I will be yielded.

$$
I=\Phi \cdot S
$$

Where, $\mathrm{S}$ is receiving area of photodiode. $\Phi$ is luminous flux.The current is proportional to the luminous flux.

The equivalent circuit of photodiode is shown in Fig.1. The circuit contains a current source related to the light which parallels to a shunt resistance and a shunt capacitance. 


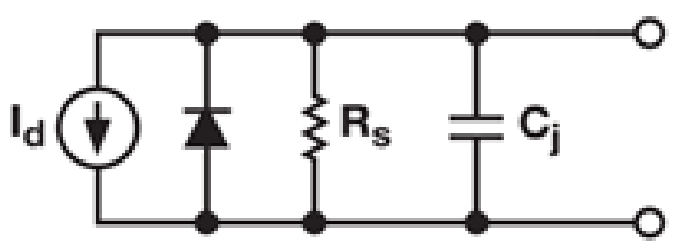

Fig.1 The equivalent circuit model of photodiode

Characteristic curves of typical photodiode is shown in Fig.2.

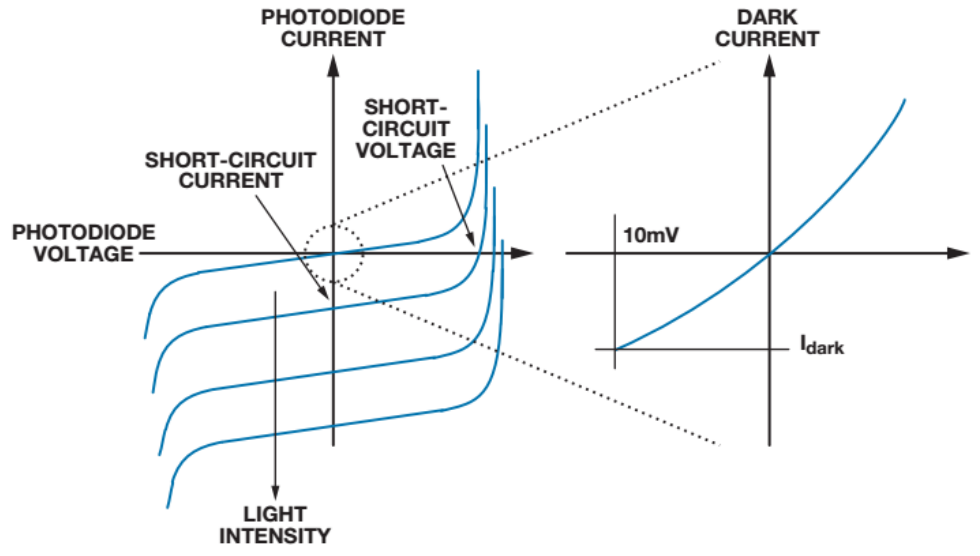

Fig.2 Characteristic curves of photodiode

The curve represents that there has no light. The current of photodiode is very weak, so the task of the photoelectric detection system is to extract the weak signal, especially to extract the weak signal covered by noise. Photoelectric detection circuit based on transimpedance amplifier realized by using transimpedance amplifier is shown in Fig.3. The pressure drop of feedback resistance RF is given as following

$$
V_{\text {OUT }}=I_{\mathrm{d}} \cdot R_{\mathrm{f}}=\Phi \cdot S \cdot R_{\mathrm{f}}
$$

The reversed-phase input of ideal amplifier is virtual ground, all current flow through feedback resistance RF. So the input bias current and the input offset voltage must be very small.

Fig.4a is the actual parameter circuit model of Fig.3. The system has two poles, one is the pole of the low frequency open loop transfer function, and the other is the closed loop pole which is caused by feedback resistance and the parasitic resistance and capacitance of photodiode. One pole causes phase shift $90^{\circ}$ of open loop transfer function, so the total phase shift is $180^{\circ}$, which is considerably lower than the open loop amplitude phase shift which the frequency strides $0 \mathrm{~dB}$. If phase margin is lack, pole has half plane in s plane (shown in Fig.4a), which must cause circuit oscillation.

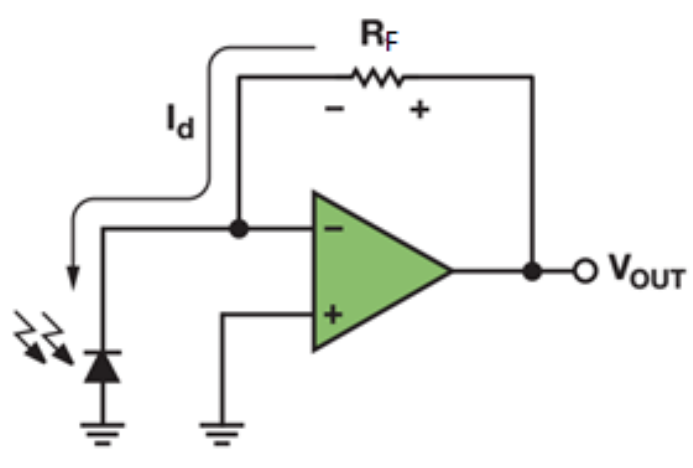

Fig.3 Photoelectric detection circuit based on transimpedance amplifier

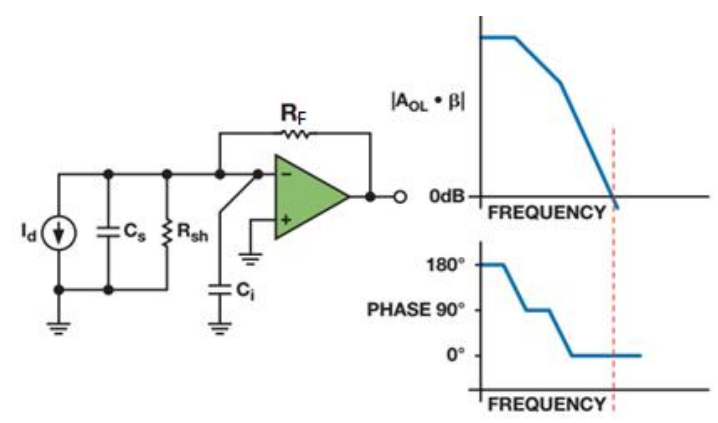

(a)

Fig.4 Frequency response model and response curves of photoelectric detection circuit based on the photodiode 
In order to ensure working stable, to let a capacitance parallel to RF, it can add a zero to the transfer function, and yields positive phase margin to ensure the stability. In order to get $45^{\circ}$ phase margin, it must satisfy the following equation

$$
C_{\mathrm{f}}=\sqrt{\frac{C_{\mathrm{sh}}+C_{\mathrm{i}}}{2 \pi R_{\mathrm{f}} f_{\mathrm{u}}}}
$$

Where, fu is the unit gain frequency of the operational amplifier, and the highest actual frequency of the system is determined by the value of $\mathrm{Cf}$.

The programmable gain amplifier can cascade to ensure the transimpedance gain of amplifier is in the linear region, or the programmable gain is realized in TIA directly to structure PGTIA, which is shown in Fig.5. A type of PGTIA is shown in Fig.6. But the on-resistance and leakage current of analog switch may result in errors, so the improved circuit is shown in Fig.7.

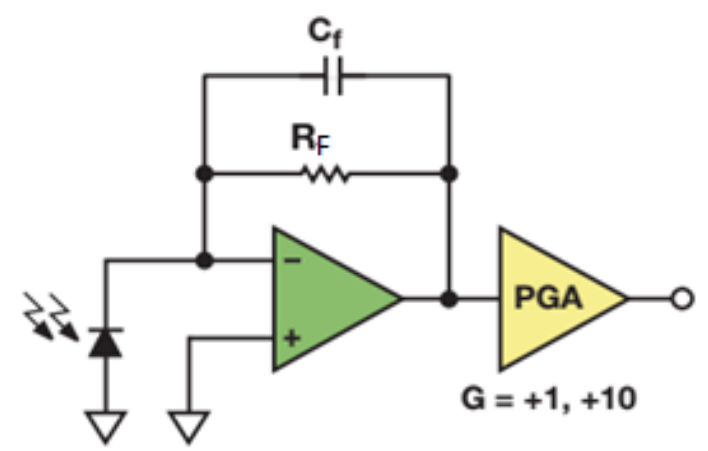

Fig.5 The first stage of TIA cascade to PGA forms PGTIA

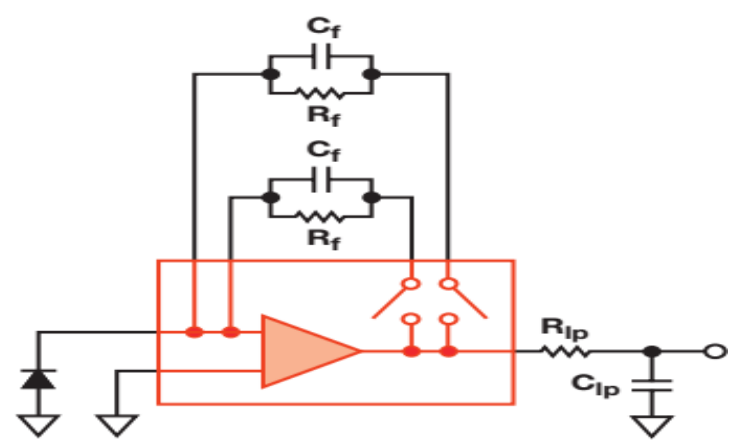

Fig.6 Programmable gain transimpedance amplifier

There are two switches in each transimpedance branch of the circuit shown in Fig.7, which avoids the above questions. Although the number of switches is double, the on-resistance of the left switches is in the feedback loop, so the output voltage depends on the current which flows through the selected resistance. The switches on the right are seemingly output impedance, the errors caused by who can be neglectable, if the amplifier drive high impedance load such as ADC driver.

The circuit shown in Fig.7 is suitable for DC and low frequency, when it is turn-off, parasitic capacitance on the switch will lead to new problems. The influence can be decreased by replacing the switches by two cascaded switches, the parasitic capacitance will be halving, but more elements are used.

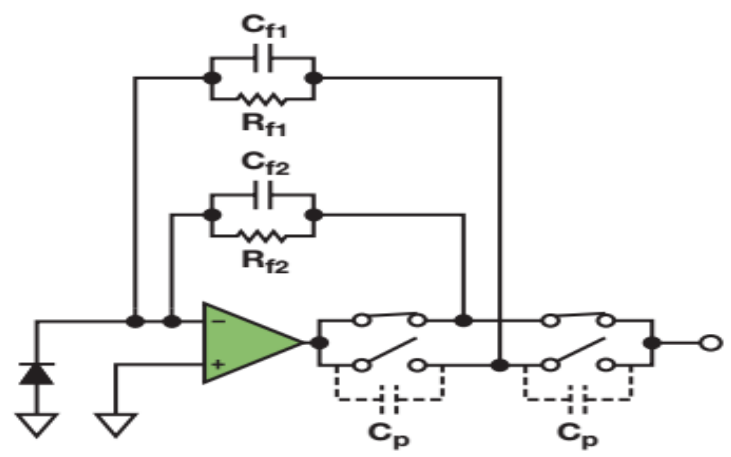

Fig.7 Programmable gain transimpedance amplifier with Kelvin switch

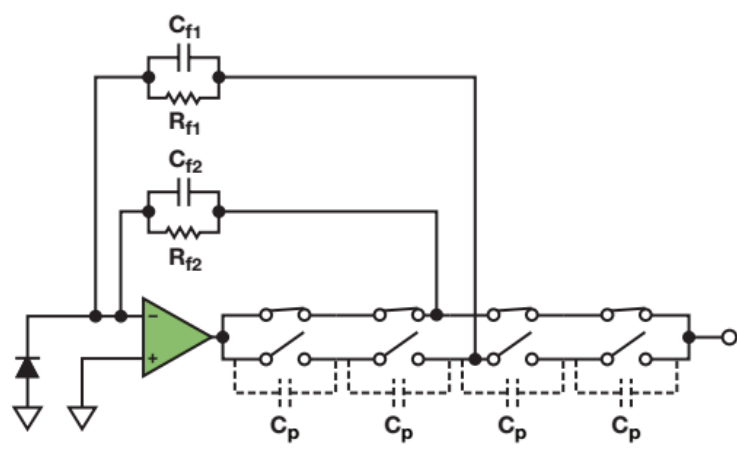

Fig.8 Increase cascaded switches to decrease total parasitic capacitance

Total feedback capacitance $=C_{f 2}+\frac{C_{p} C_{f 1}}{C_{p}+C_{f 1}}$ 
Transimpedance amplifier has three main noise sources: input voltage noise of operational amplifier, input current noise and feedback resistance noise. In addition, PGA stage is thoroughly eliminated by using programmable gain transimpedance amplifier, so the transimpedance resistance just uses its own capacitance to compensate the input capacitance of photodiode.

\section{Improved Circuit}

In addition, in order to get big output voltage and not use big feedback resistance, it is improved by using T-type feedback network. The improved circuit is shown in Fig.9. In the improved circuit, the value of the feedback resistance is not need to select too big, and the proper adjustment result of T-type feedback network can be got by selecting the radio of R1/R2. The conversion formula can be got based on kirchhoff' s law

$$
v_{\mathrm{o}}=i_{\mathrm{s}}\left[R_{\mathrm{F}}+R_{1}+\frac{R_{\mathrm{F}} R_{1}}{R_{2}}\right]
$$

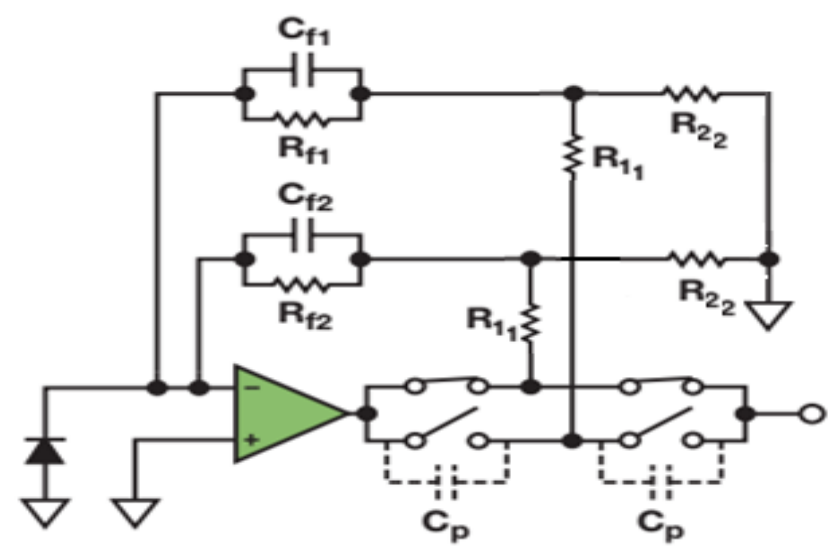

Fig.9 Improved circuit

\section{Conclusions}

Transimpedance signal detection circuit based on the photodiode is the basic component of the most photoelectric detection system. PGTIA is used in the paper, engineers can design a device to measure very big dynamic range, bandwidth and stability accurately, and select proper configuration, weigh the pros and cons, and at last, determine the detection circuit with actual index requirement.

\section{Acknowledgement}

To be explained that photodiode can be reverse connected, so the output voltage is reversed.

\section{References}

[1] B. Li, C. L. Zhao, M. W. Sun, Z. Zhou, A. Nallanathan, "SpectrumSensing for Cognitive Radios in Time-Variant Flat Fading Channels:A Joint Estimation Approach," IEEE Transactions on Communications, 2014, vol. 62, no. 8, pp. 2665-2680.

[2] B. Li, M. W. Sun, X. F. Li, A. Nallanathan, C. L. Zhao, "EnergyDetection based Spectrum Sensing for Cognitive Radios Over Time-Frequency Doubly Selective Fading Channels," IEEE Transactions on Signal Processing, vol. 63, no. 2, Jan. 2015, pp. 402-417. 
[3] MENG Haoyu, WANG Yan, WANG Cheng-wei, HU Xing-liu,FANG Ting. Design of the Weak Fluorescence Signal Detection System Based on the Principle of Lock-In Amplifier[J].Optics \& Optoelectronic Technology. 2014.12(6):89-90.

[4] B.Li, Z.Zhou, W.X.Zou, X.B.Sun, and G. L. Du, "On the efficient beam-forming training for 60 ghz wireless personal area networks," IEEE Trans. Wireless Commun., vol. 12, no. 2, Feb. 2013, pp. 504-515.

[5] B. Li, S. H. Li, A. Nallanathan, C. L. Zhao, "Deep Sensing for FutureSpectrum and Location Awareness 5G Communications," IEEE JournalSelected Areas Communications, vol. 33, no. 7, 2015, pp. 1331-1344.

[6] CHEN Zhangwei, LI Yu-he, LI Qingxiang, WANG Hang, DUAN Ruiling. The Design and Research of Prime Amplification Circuit for Photo-Detection System[J]. E1 ectrical Measurement \& Instrumentation, 2005,42(474):33.

[7] Suo jianjun. Design of an amplifying circuit on Photoelectric detection technology[J]. Practical Electronics, 2013,4:38.

[8] Li Bin. Electronic amplification system for the measurement of Laser Radar Cross Section[D]. Xi'an Electronic and Science University, 2012:46-48. 